\title{
New Opportunities for NDT Using Non-Linear Interaction of Elastic Waves with Defects
}

\author{
Igor Solodov - Daniel Döring - Gerd Busse* \\ Institute of Polymer Technology (IKT), Non-Destructive Testing (IKT-ZfP), Stuttgart University, \\ Germany
}

When material is subjected to an intense load, its stiffness changes due to elastic nonlinearity. This effect is especially pronounced in damaged materials, so that nonlinearity can be used as an indication of incipient damage. Under dynamic load, mechanical constraint between the fragments of planar defects provides gigantic nonlinearity in finite-amplitude contact vibrations. The local vibration spectra acquire a number of new frequency components which are used as signatures of damage. The experimental implementation of nonlinear NDT relies on the use of laser interferometers (Nonlinear Laser Vibrometry, $N L V)$ to detect the nonlinear waves that are generated selectively by defects. In addition, the planar defects as localized sources of nonlinear vibrations efficiently radiate nonlinear airborne ultrasound (Nonlinear Air-Coupled Emission, NACE). The frequency conversion mechanism concerned with contact nonlinearity of the defect vibrations provides an efficient generation of air-coupled higher-order ultra-harmonics, ultrasubharmonics, and combination frequencies. Both the NLV and NACE are proposed for remote scanning and high contrast defect-selective imaging in nonlinear NDT.

(C2011 Journal of Mechanical Engineering. All rights reserved.

Keywords: Nonlinear NDT, defect-selective imaging, nonlinear air-coupled emission

\section{INTRODUCTION}

Conventional ultrasonic Non-Destructive Testing (NDT) is normally based on the scattering of acoustic waves by defects leading to the amplitude and phase variations of the input signal due to the wave-defect interaction. The efficiency of the interaction depends on the size of the defects and a degradation of linear material properties (that is, a local stiffness) caused by the damage. For incipient damage, the contribution of both factors is negligible and determines unacceptably low sensitivity of the technique for NDT of this type of defects.

The nonlinear approach to NDT (NNDT) makes use of the fact that the nonlinear part of the stress-strain diagram is a sensitive indicator of the presence of defects. The dynamic nonhookean behaviour of the material is concerned with non-linear response of defects, which is related to the frequency changes of the input signal. These spectral changes are caused by anomalously nonlinear local dynamics of defects of various scale and nature. Monitoring of the local nonlinearity makes NNDT defect-selective, i.e. it reveals directly the vulnerable (faulty) areas within material or a product.

In damaged materials, the nonlinear response is provided by the Contact Acoustic Nonlinearity (CAN) [1]: strongly nonlinear local vibrations of defects due to mechanical constraint of their fragments, which efficiently generate multiple ultra-harmonics and support multi-wave interactions. Another contribution to the nonlinear spectrum comes from resonance properties of planar defects [2]. Similar to the resonance behaviour of an air bubble in a liquid, vibrations of a certain mass of material around a cracked defect are managed by reduced stiffness which provides a specific characteristic frequency of the defect and brings the nonlinear resonance scenario into elastic wave interaction with defects.

In this paper, basic mechanisms responsible for frequency conversion in the nonlinear elastic wave-defect interaction are reviewed and major features of CAN spectra are discussed. Experimental methodologies of nonlinear laser vibrometry (NLV) and nonlinear air-coupled emission (NACE) are introduced and used to study the nonlinear spectra of defects. Applications for defect-selective imaging and

*Corr. Author's Address: Institute of Polymer Technology (IKT), Non-Destructive Testing (IKT-ZfP), Stuttgart University, Pfaffenwaldring 32, 70569 Stuttgart, Germany, gerd.busse@ikt.uni-stuttgart.de 
NNDT are demonstrated for a series of hi-tech materials and industrial components.

\section{PHENOMENOLOGY OF HIGHER HARMONIC GENERATION VIA CAN}

The CAN generally manifests in a wide class of damaged materials and is caused by mechanical constraint between the fragments of planar defects which makes their vibrations extremely nonlinear. The CAN develops diversely for longitudinal and shear driving tractions which activate different mechanisms of nonlinearity.

\section{1 "Clapping" Mechanism}

Consider a pre-stressed crack (static stress $\sigma^{0}$ ) driven with longitudinal acoustic traction $\sigma_{\sim}$ (Fig. 1) which is strong enough to provide clapping of the crack interface. The clapping nonlinearity is due to asymmetrical dynamics of the contact stiffness: the latter is, apparently, higher in a compression phase (due to clapping) than that for tensile stress when the crack is assumed to be supported only by edge-stresses.

Such behavior of a clapping interface can be approximated by a piece-wise stress $(\sigma)$-strain (ع) relation [3]:

$$
\sigma=C[1-H(\varepsilon)(\Delta C / C)] \varepsilon,
$$

where $H(\varepsilon)$ is the Heaviside unit step function; $\Delta C=\left[C-(d \sigma / d \varepsilon)_{\varepsilon>0}\right]$, and $C$ is the intact material (linear) stiffness.

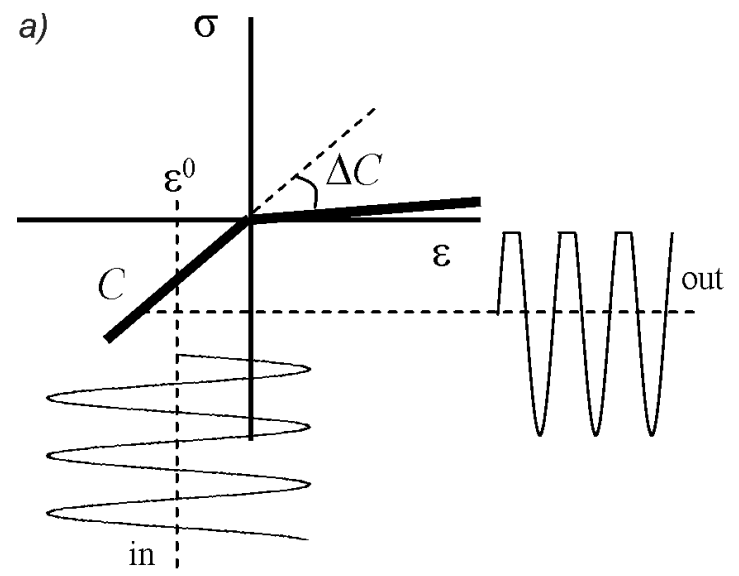

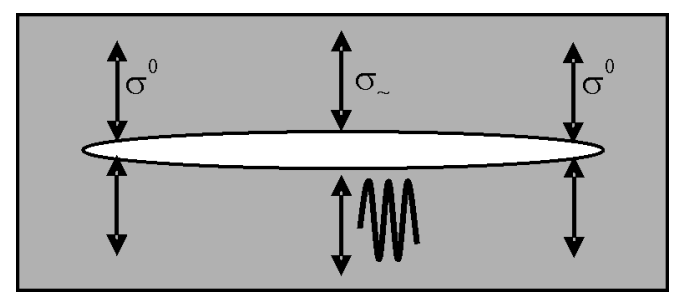

Fig. 1. Model of a clapping crack

The bi-modular pre-stressed contact driven by a harmonic acoustic strain $\varepsilon(t)=\varepsilon_{0} \cos v t$ is similar to a "mechanical diode" and results in a pulse-type modulation of its stiffness $C(t)$ and a half-period rectified output (Fig. 2). Since $C(t)$ is a pulse-type periodic function of the driving frequency $v$ (Fig. 2b), the nonlinear part of the spectrum induced in the damaged area $\left(\sigma^{N L}(t)=\Delta C(t)^{\cdot} \varepsilon(t)\right)$ contains a number of its higher harmonics $n v$ (both odd and even orders) whose amplitudes are modulated by the sincenvelope function [4]:

$$
\begin{gathered}
A_{n}=\Delta C \Delta \tau \varepsilon_{0}[\sin c((n+1) \Delta \tau)- \\
-2 \cos (\pi \Delta \tau) \sin c(n \Delta \tau)+\sin c((n-1) \Delta \tau)],
\end{gathered}
$$

where, $\Delta \tau=\tau / T \quad \tau=(T / \pi) \operatorname{Arc} \cos \left(\varepsilon^{0} / \varepsilon_{0}\right) \quad$ is the normalized modulation pulse length.

The spectrum of the nonlinear vibrations (2) is illustrated in Fig. 3a and contains a number of both odd and even higher harmonics arising simultaneously as soon as $\varepsilon>\varepsilon^{0}$ (threshold of clapping). The sinc-modulation in Eq. (2) is amplitude dependent: as the wave amplitude

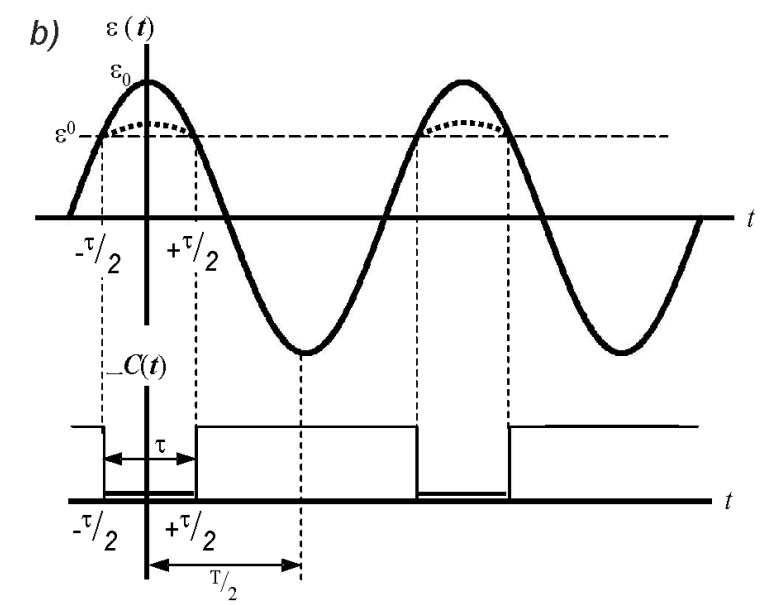

Fig. 2. a) Mechanical diode model, b) stiffness modulation and waveform distortion 

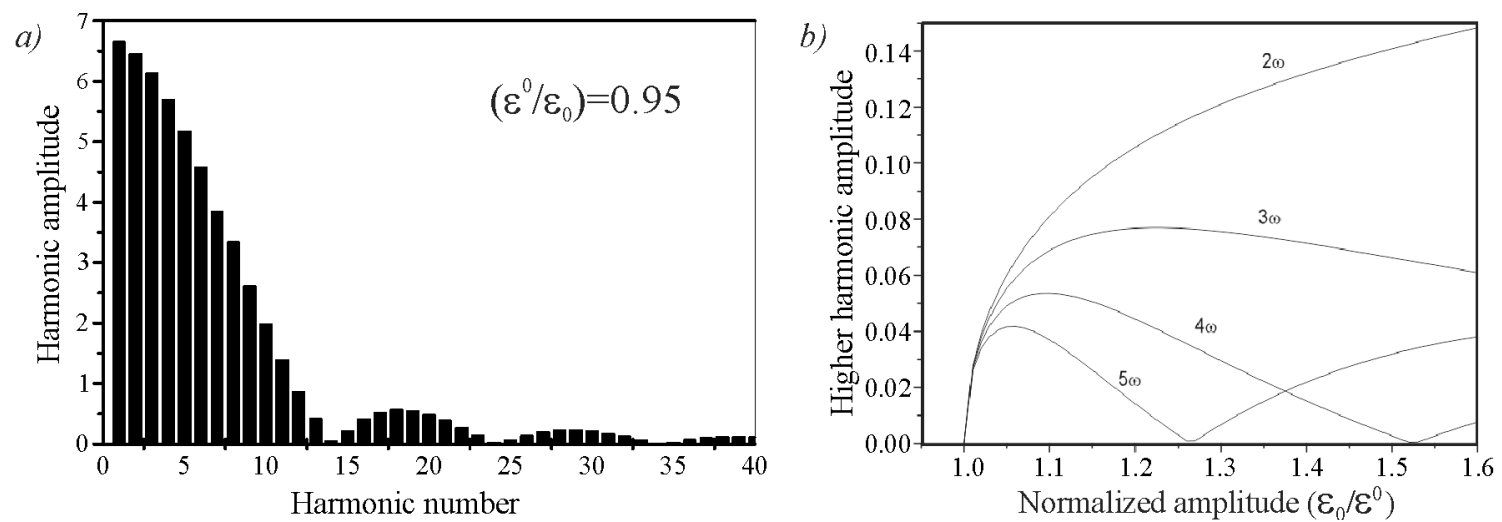

Fig. 3. a) CAN higher harmonic spectrum, b) dynamic characteristics

$\varepsilon_{0}$ increases, $\tau$ grows from 0 to $T / 2$. This affects dynamic characteristics of the higher harmonics (Fig. 3b) and provides their amplitude oscillations for $\varepsilon_{0}>\varepsilon^{0}$ due to the $\Delta C(t)$ spectrum "compression" effect.

\subsection{Nonlinear Friction Mechanisms [4]}

For a shear wave drive, the surfaces of the contact interface are mechanically coupled by the friction force caused by the interaction between asperities (Fig. 4). If the driving amplitude is small enough, the interface shear motion is constrained by the interaction between neighboring asperities which prevents the contact surfaces from sliding (micro-slip mode). The mechanical diode model for the micro-slip motion is shown in Fig. 5a and demonstrates a step-wise increase in tangential stiffness as the neighboring asperities interact. This interaction is independent of the direction of shear motion and causes stiffness variation twice for the input signal period (Fig. 5b). Similar to the hysteresis case, such a constraint introduces a symmetrical nonlinearity and provides only odd harmonic generation. Similar to the clapping mechanism, their amplitudes are sinc-modulated due to pulse-type stiffness variation [5] (Fig. 6):

$$
\begin{aligned}
A_{2 N+1}= & 2 \Delta C \varepsilon_{0}\left(\frac{\tau}{T}\right) \\
& \left\{\operatorname{sinc}\left(\frac{2 N \tau}{T}\right)+\operatorname{sinc} \frac{2(N+1) \tau}{T}\right\}
\end{aligned}
$$

and exhibit a similar non-power dynamics.

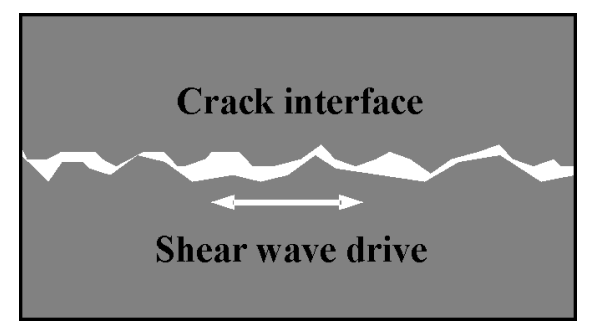

Fig. 4. Crack interface in shear drive

When the amplitude of tangential traction is greater than the contact static friction force, the micro-slip motion changes for sliding. An oscillating shear wave drive is accompanied by a cyclic transition between static and kinematic friction (stick-and-slide mode), so that the contact stress-strain relation follows a hysteretic loop [4]. The contact tangential stiffness changes symmetrically (independent of the direction of motion) between the static (for a stick phase) and dynamic values (in slide phase) twice over the input strain period which provides odd higher harmonics generation. Similarly, the CAN features sinc-spectrum modulation and non-power dynamics.

\section{EXPERIMENTS ON HIGHER HARMONIC GENERATION BY DEFECTS}

Some experimental results which confirm the CAN spectral properties are presented in Figs. 7 to 9 . In the experiments, the methodology of NLV [6] that includes an intense CW acoustic excitation (flexural waves) in the kHz-frequency range combined with a laser probing of the spectra 
a)

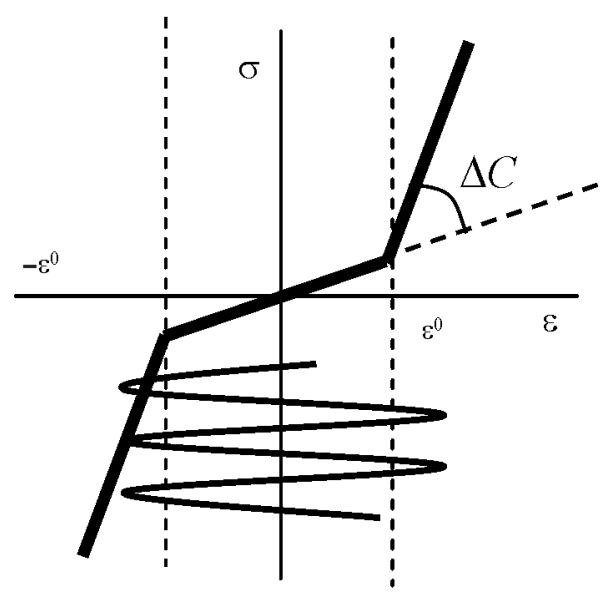

b)

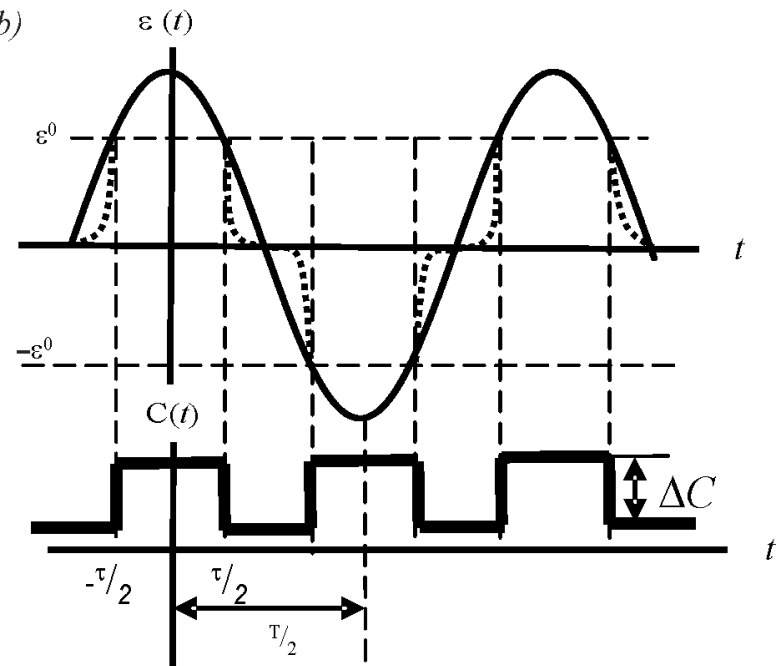

Fig. 5. a) Mechanical diode model, b) stiffness modulation and waveform distortion in micro-slip mode

and temporal vibration patterns was used. The specimen of multi-ply carbon-fibre-reinforced plastic (CFRP) with an impact damage area (Fig. 7a) was measured.

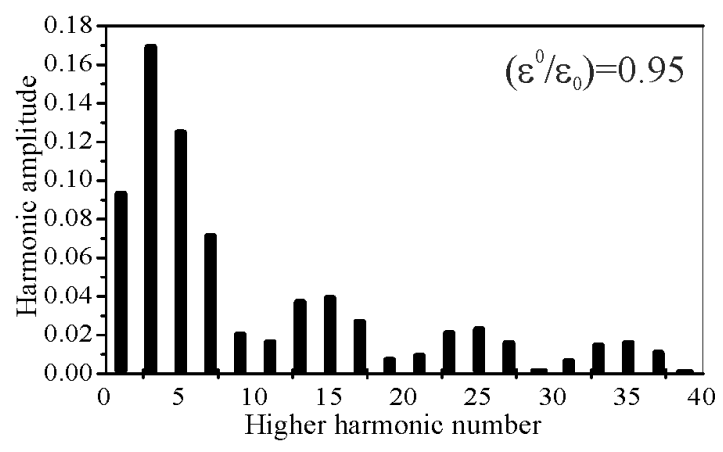

Fig. 6. Sinc-modulated odd higher harmonic CAN spectrum in micro-slip mode
The temporal vibration pattern measured outside the damage is a sinusoidal signal with a negligible distortion (Fig. 7b). The waveform changes dramatically in the damaged area: the half-period rectified signal is observed (Fig. $8 b$ ) in perfect agreement with the diode model predictions for the clapping CAN mechanism. The higher harmonic spectrum (Fig. 8a) exhibits an evident sinc-amplitude modulation which is also typical for the clapping contact.

The mechanism of friction nonlinearity was found to prevail in wood which is a natural fibre-reinforced composite. In intact wood, the nonlinear spectrum averaged over the specimen surface exhibits an evident odd harmonic domination (Fig. 9a). Then, the specimen was damaged (crack made by cutting), and the spectrum measured in the neighborhood of the
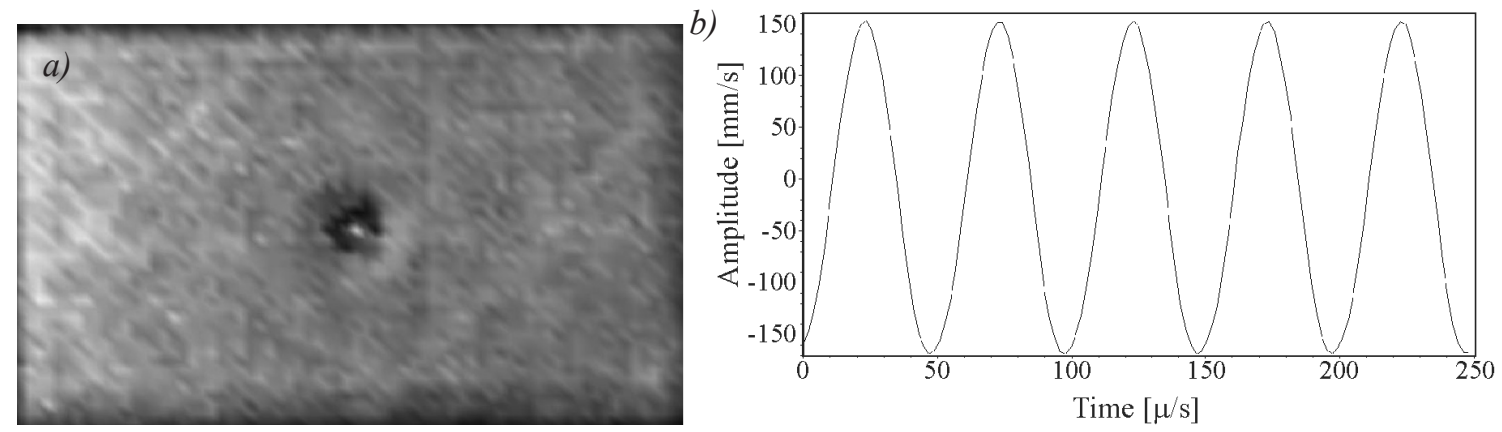

Fig. 7. a) CFRP-specimen with impact damage area, b) temporal vibration pattern outside damage 

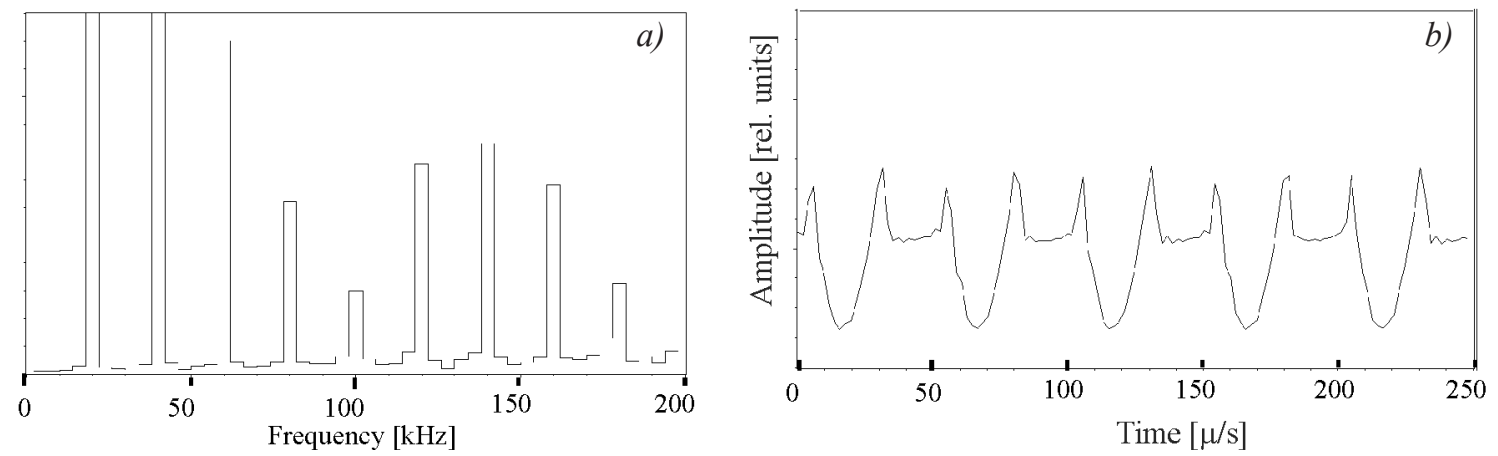

Fig. 8. a) Higher harmonic spectrum, b) temporal waveform pattern in the damaged area
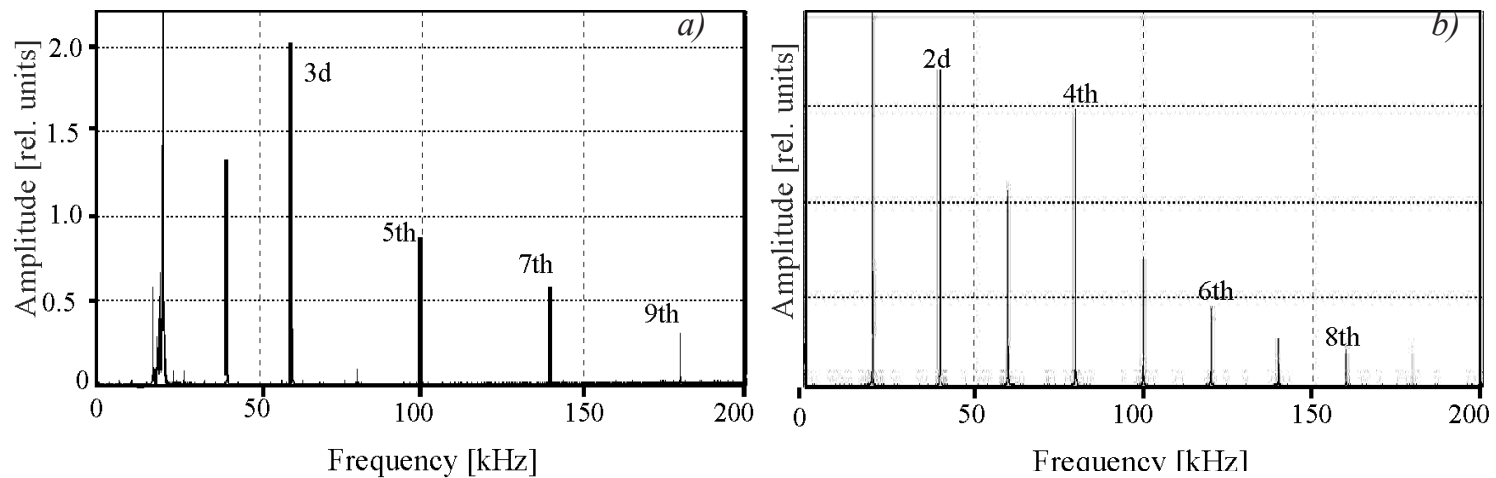

Fig. 9. a) Odd, b) odd/even higher harmonic spectrum in intact and damaged wood respectively

crack acquires even higher harmonics (Fig. 9b) obviously generated due to contact clapping in the crack. The change of type nonlinearity enables, therefore, the use of the harmonic signature for discerning and imaging flaws in wood and wood composites [7].

\section{ULTRA-SUBHARMONIC AND ULTRA- FREQUENCY PAIR NONLINEAR MODES}

\subsection{Phenomenology}

In addition to the higher harmonic generation, the experiments [2] and [8] also revealed different scenarios of CAN dynamics, which expand considerably nonlinear spectra of cracked defects. These scenarios exhibit forms of dynamic instability, i.e. an abrupt change of the output for a slight variation of the input parameters. To illustrate the feasibility of the new nonlinear vibration modes and ascertain their basic spectral patterns, it is assumed that the crack exhibits both resonance and nonlinear properties and is thus identified as a nonlinear oscillator [2]. Its characteristic frequency $\left(\omega_{0}\right)$ is determined by a linear stiffness and an associated mass of the material inside the damaged area (Fig. 10). The contact nonlinearity is introduced as displacement $(X)$ dependent nonlinear interaction force $F^{N L}(X)$. The driven vibrations (driving force $\left.t(t)=f_{0} \cos v t\right)$ ) of the nonlinear oscillator are found as a solution to the nonlinear Eq.:

$$
\ddot{X}+\omega_{0}^{2} X=f(t)+F^{N L}(X) .
$$

In the second order of the perturbation approach $F^{N L} \sim \cos \left(v-\omega_{0}\right) t$ that accounts for the interaction between driving and natural frequency vibrations. If $v-\omega_{0} \approx \omega_{0}$, the resonance increase in the output at $\omega_{0} \approx v / 2$ is observed (subharmonic generation). The higher-order terms correspond to the frequency relation $m v-n \omega_{0}$ that provides resonance output at $\omega_{0} \approx m v /(n+1)$. For $n=1$, the crack generates ultra-subharmonics (USB) of 
the second order $m v / 2$; the higher order USB correspond to the higher values of $n$.

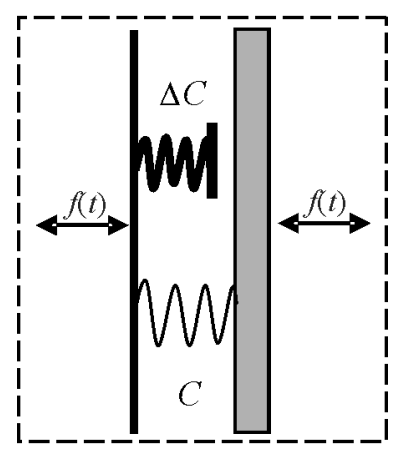

Fig. 10. Resonance model of a clapping crack

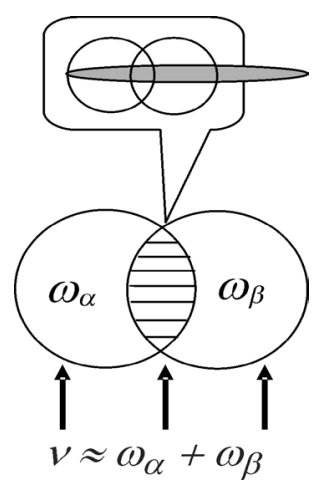

Fig. 11. Crack as a set of coupled nonlinear oscillators

In reality, a damaged area has a more complicated structure that can be conceived as a set of coupled nonlinear oscillators (a pair of those with normal frequencies $\omega_{\alpha}$ and $\omega_{\beta}$ is shown in Fig. 11). If the frequency of the driving acoustic wave is $v \approx \omega_{\alpha}+\omega_{\beta}$, the difference frequency components $v-\omega_{\alpha} \approx \omega_{\beta}$ and $v-\omega_{\beta} \approx \omega_{\alpha}$ provide cross excitation of the coupled oscillators. It results in a resonant generation of the frequency pair $\omega_{\alpha}, \omega_{\beta}$ centred around the subharmonic position. The higher-order nonlinear terms in Eq. (4) expand the CAN spectrum, which comprises multiple ultra-frequency pairs (UFP) centred around the higher harmonics and USB [2].

USB and UFP belong to the class of the instability modes and can be interpreted, respectively, as a half-frequency and combination frequency decay of a high-frequency phonon (driving frequency signal). The resonance growth of these modes is possible only if the input excitation exceeds a certain threshold; it is then affected by the amplitude and frequency instability and hysteresis [9]. The resonance instability manifests itself in the avalanche-like amplitude growth beyond the input threshold. The reverse amplitude excursion results in bistability: the input amplitudes for the up and down transitions are different (amplitude hysteresis). Such a dynamics is a distinctive signature of the nonlinear acoustic phenomena associated with nonlinear resonance.

\subsection{Experimental Observation of USB and UFP Spectra}

Examples of the USB and UFP spectra observed in damaged materials by using NLV are shown in Figs. 12 and 13.

The USB spectrum in Fig. 12 is measured in a cracked area of a polystyrene plate driven at $\sim 1.3 \mathrm{kHz}$ with a shaker. The higher harmonic pattern changes abruptly for the USB spectrum as the driving amplitude grows beyond a certain threshold value. The "wavy" amplitude modulation in Fig. 12 indicates the involvement of the CAN mechanisms in USB generation.

Fig. 13 shows a section of the nonlinear spectrum measured in a glass-fibre reinforced composite (GFRP) with an impact damage for a $20 \mathrm{kHz}$ excitation beyond the UFP-threshold. The positions of the second $(40 \mathrm{kHz})$ and third $(60$ $\mathrm{kHz}$ ) harmonics as well as ultra-subharmonics (50 and $70 \mathrm{kHz}$ ) can be clearly identified. The UFP lines are centred around the USB positions and distanced by $\Delta \cong 1.2 \mathrm{kHz}$. The UFP signals with larger $\Delta$ and smaller amplitude are also seen in Fig. 13.

The dynamic properties of the nonlinear resonance modes are illustrated in Fig. 14a.

The figure shows the amplitude of the $3 \omega / 2$-subharmonic wave generated in the reflection of $30-\mathrm{MHz}$ acoustic waves from a crack in $\mathrm{LiNbO}_{3}$ crystal [8], as a function of the input voltage. The step-like thresholds $\left(\left(V_{I N}\right)_{1} \geq\right.$ $\left.3 \mathrm{~V} ;\left(V_{I N}\right)_{2} \geq 4 V\right)$ followed by the stable plateaus can clearly be seen. The sharp increase at the thresholds confirms a transition into the instability region where the avalanche-like development of nonlinear oscillations takes place. The hysteresis 


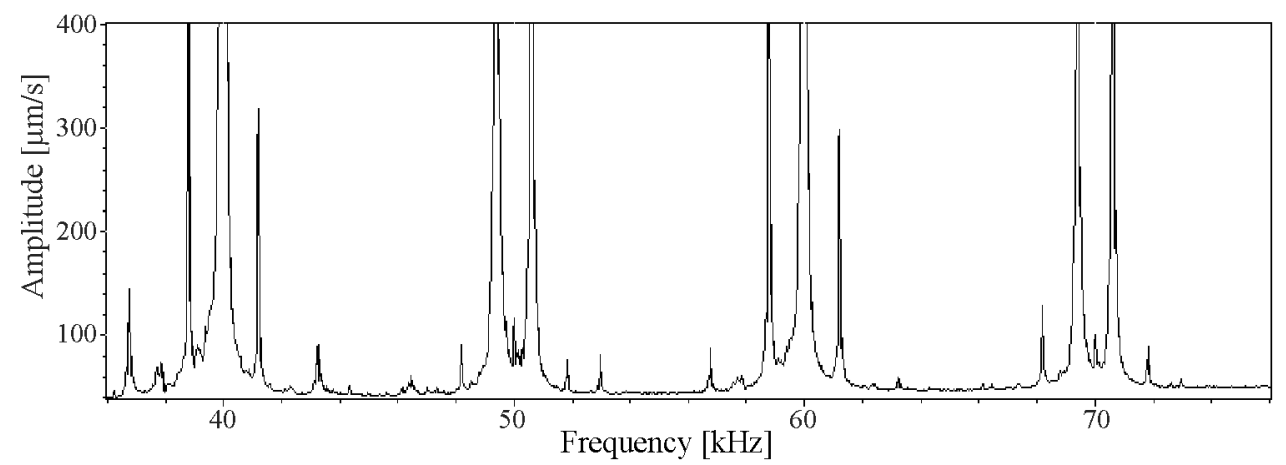

Fig. 12. USB spectrum in cracked area of polystyrene plate; the arrow indicates driving frequency

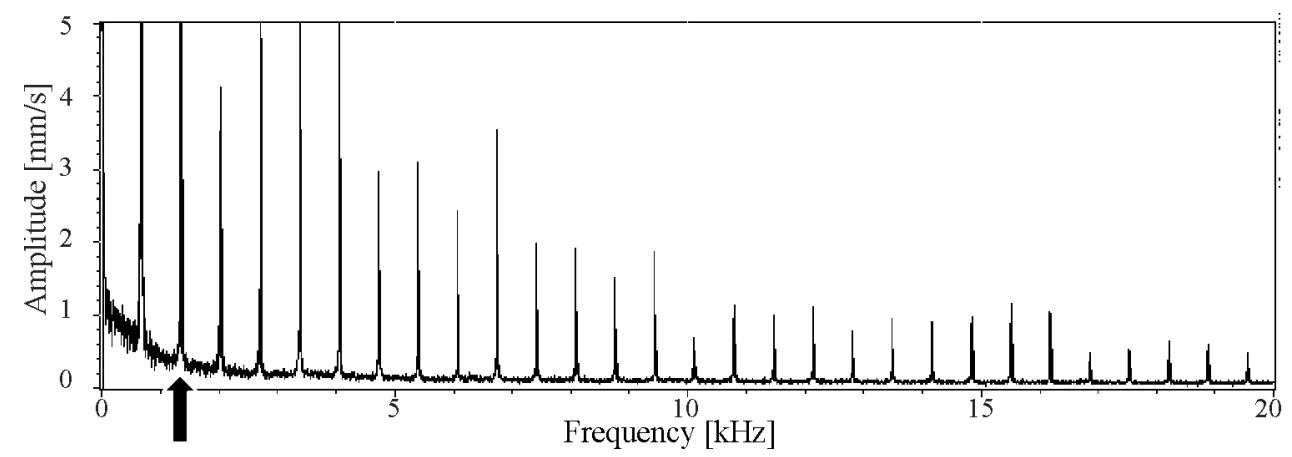

Fig. 13. UFP spectrum in impact damaged area of GFRP-specimen;driving frequency is $20 \mathrm{kHz}$
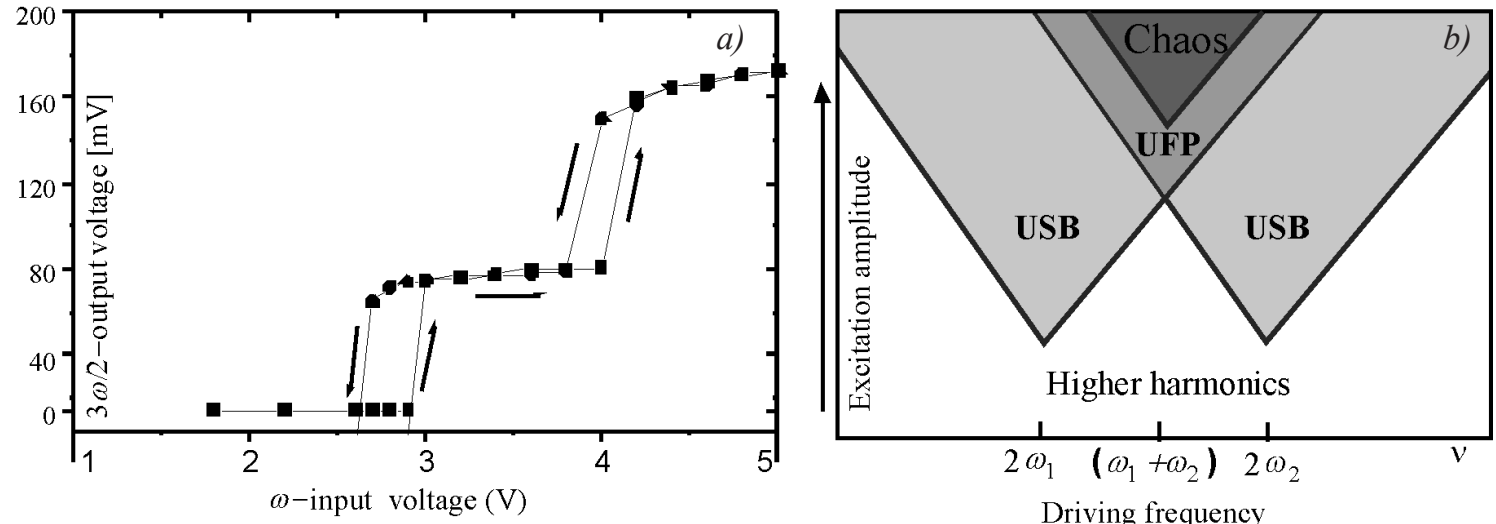

Fig. 14. a) Threshold and bistability of USB mode, b) schematic of nonlinear dynamics of a cracked defect presented as a pair of coupled nonlinear oscillators

of the curves in Fig. 14a is the evidence of bistability in the crack signature [8].

The experimental results on the nonlinear dynamics of fractured flaws are summarized schematically in Fig. 14b for a defect represented by a pair of coupled oscillators (normal frequencies $\omega_{1}$ and $\omega_{2}$ ) [10]. At low amplitudes of the driving excitation (frequency $v$ ), the nonlinear spectrum follows the non-resonant scenario of the previous section and comprises the higher harmonic $(\mathrm{HH})$ and the wave modulation (WM) frequency components. As the input amplitude exceeds the threshold value, resonance instability generally results in the activation of the USB components first. The threshold amplitude depends on the driving frequency: a minimal 
threshold requires frequency matching to the main subharmonic resonance $(v=2 \omega)$. The frequency zones for the USB generation expand readily as the excitation amplitude increases. A further increase of acoustic excitation above a given threshold gives rise to the UFP spectra. A direct transition from the non-resonance modes to the UFP-instability is also possible when the sumfrequency resonance matching conditions are satisfied. The $V$-shaped zones in Fig. 14 are typical of parametric resonance modes [9] and indicate that the frequency matching is not required for high driving amplitudes. Finally, the multiple UFP bring the system to a quasi-continuous spectrum, which indicates a build-up of chaotic vibrations.

\section{CAN APPLICATIONS IN NDT: NLV FOR DEFECT-SELECTIVE IMAGING}

The nonlinear spectra discussed above are produced locally in the damaged area while an intact part of material outside the defects vibrates linearly, i.e. with no frequency variation in the output spectrum. Thus, nonlinear defects are active sources of new frequency components rather than passive scatterers in conventional ultrasonic testing. This makes nonlinearity a defect-selective indicator of damage presence and development. The high localization of nonlinear spectral components around the origin is a basis for nonlinear imaging of damage.

The NLV [6] uses a sensitive scanning laser interferometer for detecting nonlinear vibrations of defects. The excitation system includes piezo-stack transducers operating at 20 and $40 \mathrm{kHz}$. After a 2D-scan and FFT of the signal received, the $\mathrm{C}$-scan images of the sample area are obtained for any spectral line within the frequency bandwidth of $1 \mathrm{MHz}$.

\subsection{Higher Harmonic Imaging}

Higher harmonics in damaged materials are readily generated at reasonably small input amplitudes (strain $\sim 10^{-7}$ to $10^{-6}$ ) (Fig. 14b), so that even internal or other low-amplitude vibration sources provide enough energy for the higher harmonic imaging.

The internal excitation activates a clapping mechanism of higher harmonic generation in a number of mechanical units which use gear boxes and push-pull components. In many cases, the presence of clapping may indicate a faulty part of an instrument. Fig. 15 [11] illustrates the feasibility a)

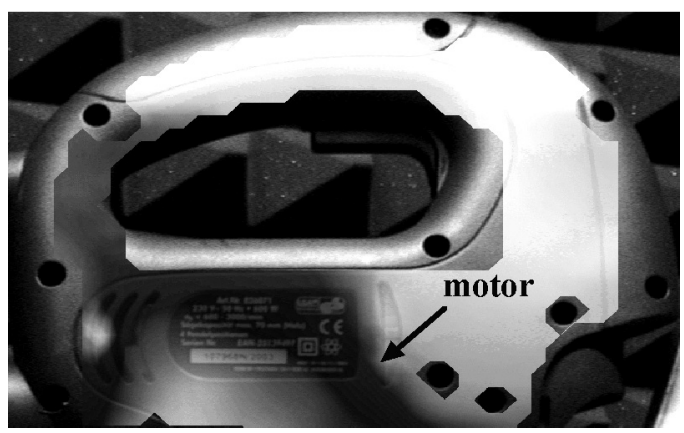

b)

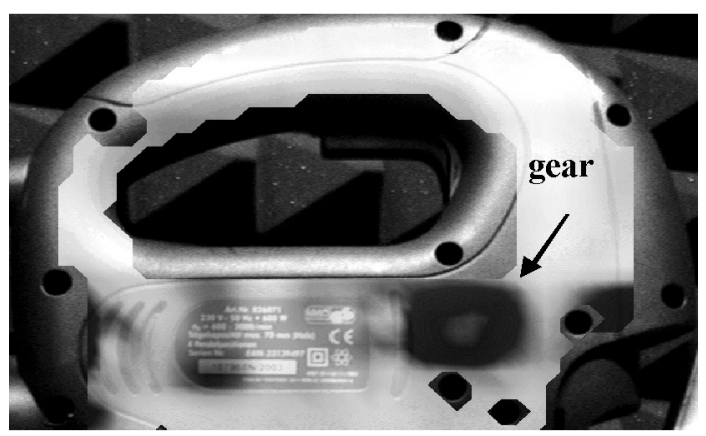

Fig. 15. a) Linear and b) third harmonic NLV-images of saw-cutting tool

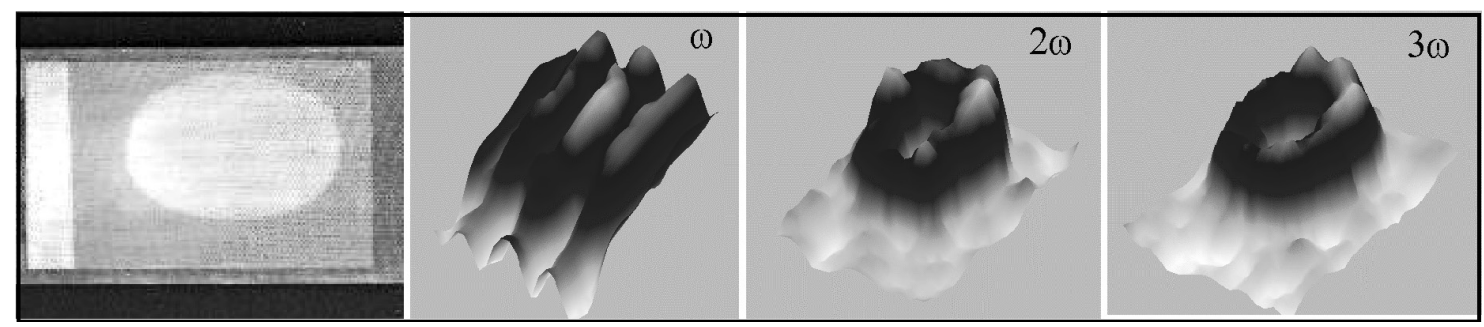

Fig. 16. Fundamental frequency ( $\omega)$ and higher harmonic imaging of a delamination in a "smart" structure 
of detection of the clapping components in a commercial saw-cutting tool. The 3D harmonic image of the motor rotation frequency clearly locates the position of the worm-gear transmission (Fig. 15b) which moves in a nonlinear (clapping) way.

Fig. 16 shows imaging results for an oval delamination on top of a piezo-actuator embedded into a glass fibre-reinforced composite (GFRP). Such "smart" structures are likely to be used for active structural health monitoring of aerospace components. The actuator itself was used as an internal excitation source fed with a few volt input. The higher harmonic images selectively reveal the boundary ring of the delamination where clapping and rubbing of the contact surfaces are, apparently, expected. On the contrary, the driving frequency $(50 \mathrm{kHz})$ image indicates only a standing wave pattern over the area of the actuator.

A flexible operation in the higher harmonic mode can be achieved even for large industrial parts by using portable ultrasonic transducers. The example in Fig. 17 shows nonlinear imaging of a fatigue crack in a riveted aviation component. The $20-\mathrm{kHz}$ excitation was implemented with "dry-contact" portable piezoelectric transducers attached to the specimen by vacuum suction.

\subsection{Nonlinear Imaging in USB- and UFP-Modes [10]}

The input acoustic power for the USB- and UFP-modes is somewhat higher than that for the higher harmonic experiments. In practical terms, this requires an acoustic intensity of a few $W / \mathrm{cm}^{2}$ in the high-MHz-frequency range and the driving amplitudes of $\mu \mathrm{m}$-scale in the low-kHz range. A strong increase of the instability modes beyond the threshold leads to distinctive nonlinear spectra with multiple USB- and UFP-components located exclusively in damaged areas. A few examples of nonlinear NDE using these modes are given below to demonstrate their applicability and superior performance in cases where the data obtained by non-resonant modes are insufficient.

Fatigue loads in metals (rotors, turbines, etc.) cause minute cracks of micro-meter scale which may gradually develop into major cracking and initiate an abrupt material fracture. Linear ultrasound is virtually unable to detect a fatigue

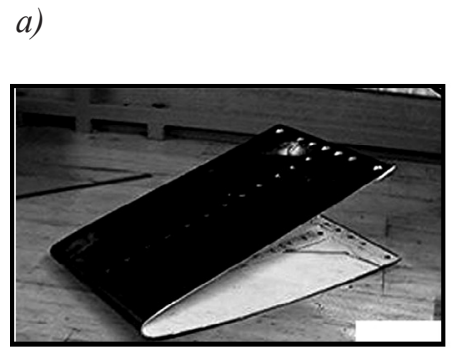

b)

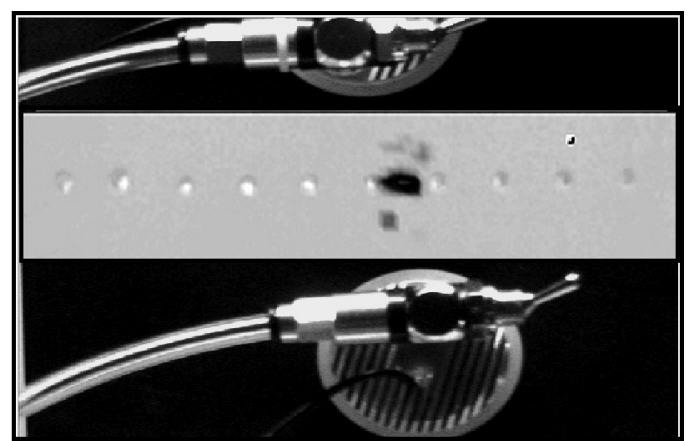

Fig. 17. a) Higher harmonic imaging of a fatigue crack in aviation component, b) excitation with portable ultrasonic transducers

a)

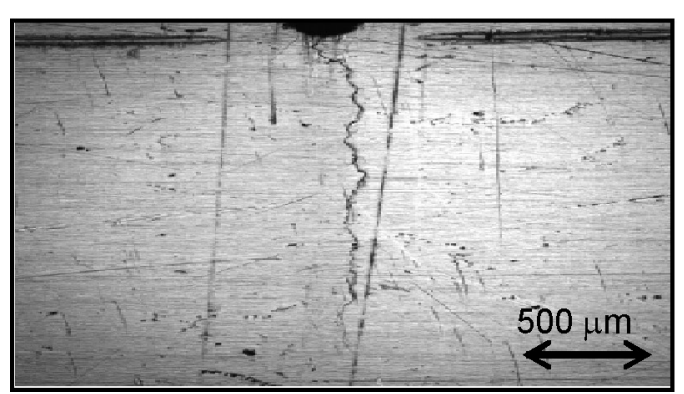

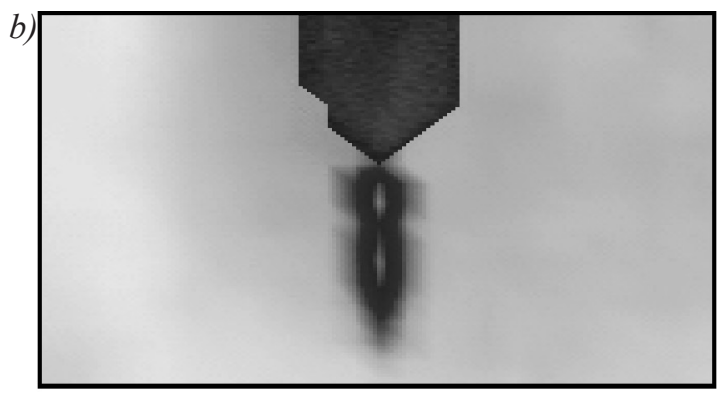

Fig. 18. a) crack photo, b) USB-image of 5 um-wide fatigue crack in Ni-base super-alloy 
crack at the early stage of their development. Examples of nonlinear imaging of fatigue induced micro-flaws and degradation of metal microstructure using the USB-modes are given in Figs. 18 and 19.

Fig. 18 shows fatigue cracking produced by cyclic loading in Ni-base super-alloys. Such a crack of $\sim 1.5 \mathrm{~mm}$ length, with average distance between the edges of only $\approx 5 \mu \mathrm{m}$, is clearly detected in the USB-image $(7 v / 2)$, whereas traditional linear NDE by using slanted ultrasonic reflection failed to work with such small cracks.

Plastic deformation is known to change the metal micro-structure by generating clusters of dislocations, which are the forerunners of micro-cracking. The feasibility of NNDE for such delicate defects in the USB-mode is illustrated in Fig. 19 for a steel auto-component (diameter $0.8 \mathrm{~cm}$; length $6.5 \mathrm{~cm}$ ) subject to $6 \%$ tensile deformation. Some necking initiated in its central part indicates that the plastic deformation was mostly concentrated in its central part. The nonlinear image reveals the area of deteriorated material properties by an evident increase in the USB amplitude.

Fiber reinforced composites constitute a class of hi-tech engineering materials whose application area is rapidly expanding in aerospace and automotive industries. Fibre metal laminates

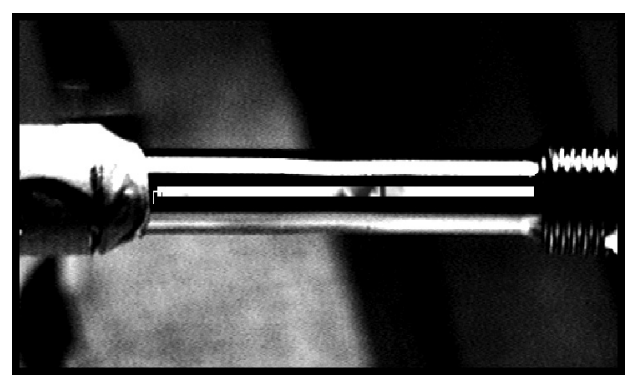

Fig. 19. USB-image of plastic deformation area in a steel component

are new materials with excellent tolerance to impact, corrosion and lightning stroke, low flammability and low weight. Fig. 20 displays an example of NNDE of such an advanced material for aircraft industry: glass fibre reinforced aluminium laminate (Glare $\left.{ }^{\circledR}\right)$. More specifically, it shows the USB-images of a Glare ${ }^{\circledR}$ plate with two inserted circular Teflon-foils to simulate local delaminations. In the images, the defect can be recognized fairly well, and the quality is enhanced for the higher orders of the USB. We believe that the latter is associated with a peculiar distribution of subharmonics over the delamination area and stronger acoustic dissipation outside the defect at higher frequencies.
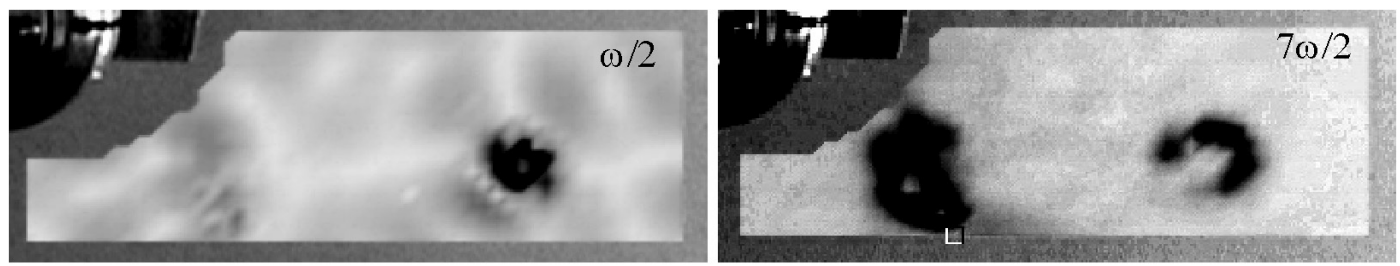

Fig. 20. USB-images of a pair of simulated delaminations in a Glare ${ }^{\circledR}$ plate
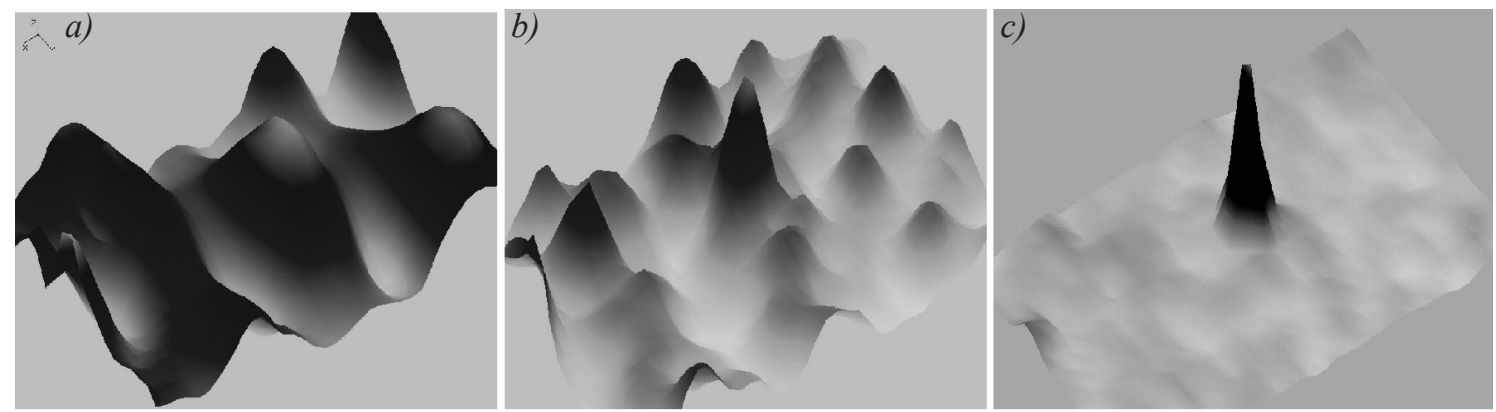

Fig. 21. a) Nonlinear imaging of impact damage in GFRP, b) linear (20 kHz-image), c) 5th harmonic image UFP - image 
Mechanical impacts in multi-ply composites produce a fracture which is a combination of matrix-fibre debonding, cracking, and delaminations. Such a combination of fractured defects makes the impact area strongly nonlinear and, normally, all nonlinear modes can be traced in the spectra observed. Similarly to all nonlinear modes discussed, the UFP-components generally display a strong spatial localization around the defects and are applicable for the detection of damage. The benefit of the UFP-mode is illustrated in Fig. 21 for a 14-ply epoxy based glass-fiber reinforced composite (GFRP) with a 9.5 J-impact damage. The linear image taken at the driving frequency of $20 \mathrm{kHz}$ reveals only a standing wave pattern over the whole sample (Fig. 21a). The higher harmonic image is also corrupted by the standing wave pattern (centre), whereas the image at the first UFP-side-lobe of the $10^{\text {th }}$ harmonic of the driving frequency $(198.8 \mathrm{kHz})$ yields a clear indication of the damaged area (Fig. 21b).

An example of nonlinear monitoring of macro-defects in constructional materials is shown in Fig. 22b for a slab of GFR-concrete $(15 \times 30 \times 1.5 \mathrm{~cm})$. Production technology of this material often suffers from internal delaminations induced by fibre-matrix debonding. For an intense $20 \mathrm{kHz}$-excitation, multiple UFP-components were measured in vibration spectra which clearly indicate the delamination areas (dark, Fig. 22a).

\section{DEFECT-SELECTIVE IMAGING VIA NONLINEAR AIR-COUPLED EMISSION (NACE)}

The scanning laser vibrometer suffers from variation of optical reflectivity; e.g. the measurements fail in damaged areas with a particularly strong scattering of laser light. Our experiments have demonstrated that planar defects as localized sources of nonlinear vibrations efficiently radiate nonlinear airborne ultrasound. Such a nonlinear air-coupled emission (NACE) is proposed as an alternative (and in many cases superior) methodology to locate and visualize defects in NNDE [12].
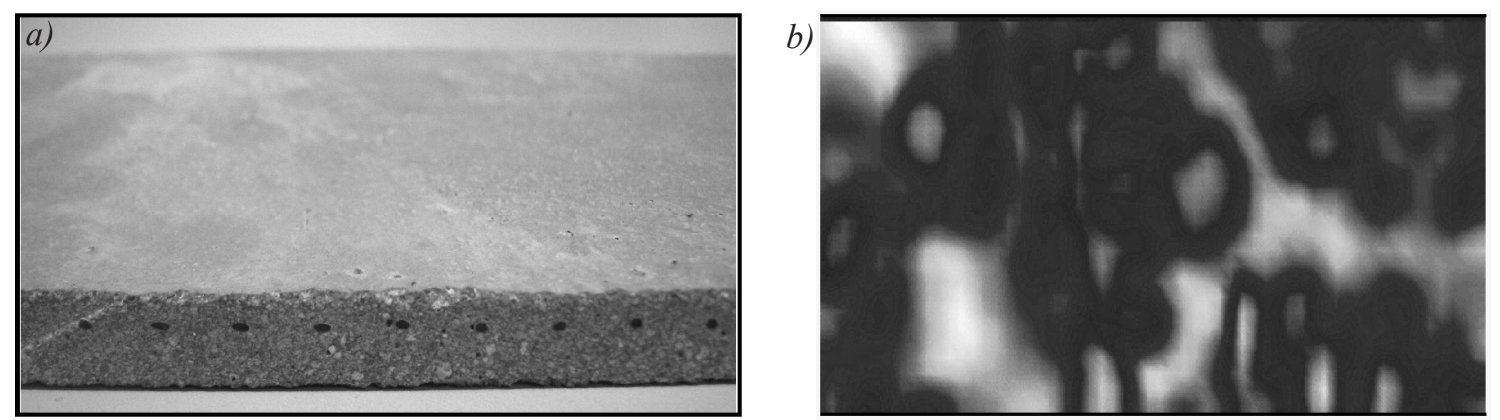

Fig. 22. UFP-image; b) of delamination area (dark) in a) GFR-concrete slab

a)

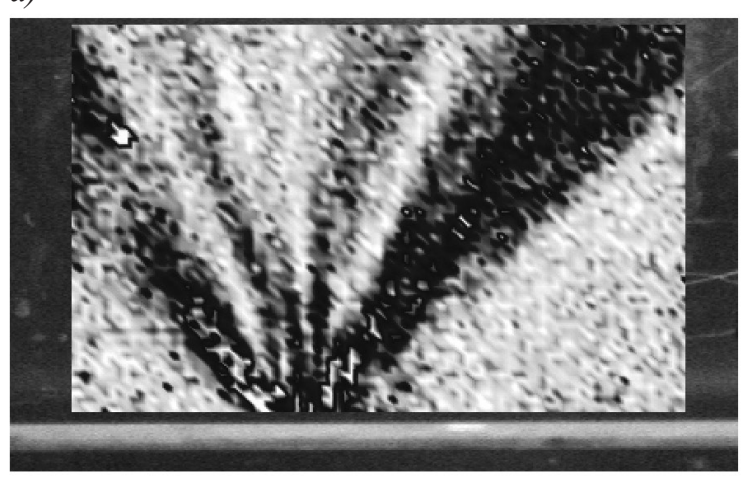

b)

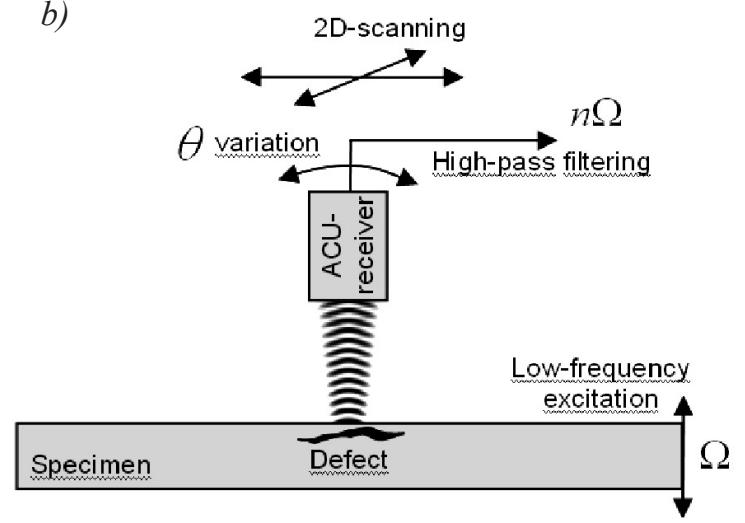

Fig. 23. a) Third harmonic air-borne radiation from delamination area, b) set-up for NACE NNDE 

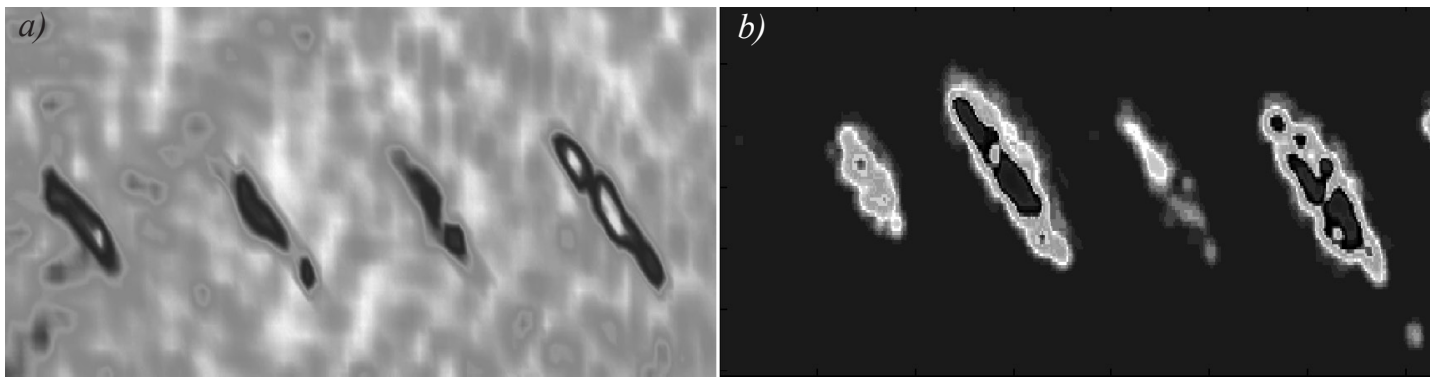

Fig. 24. Nonlinear imaging of an impact induced damage in multi-ply (+450; -450) GFR-plate: a) laser vibrometry (second harmonic image), b) NACE (9th-11th) higher harmonic image
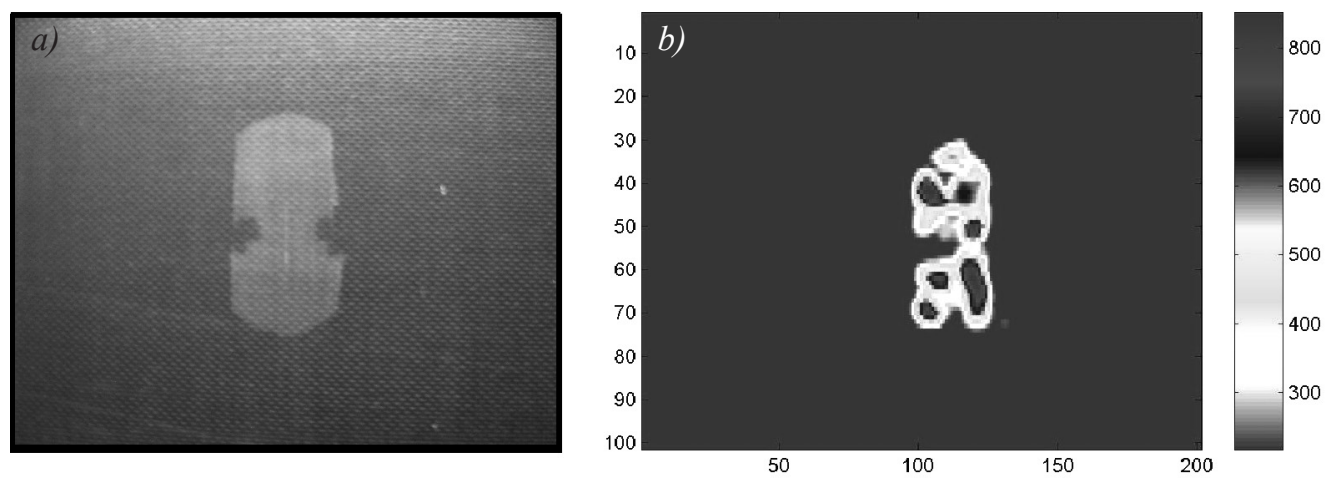

Fig. 25. NACE image (b) of $5 \times 15 \mathrm{~mm}$ delamination (a) in GFRP multi-ply plate
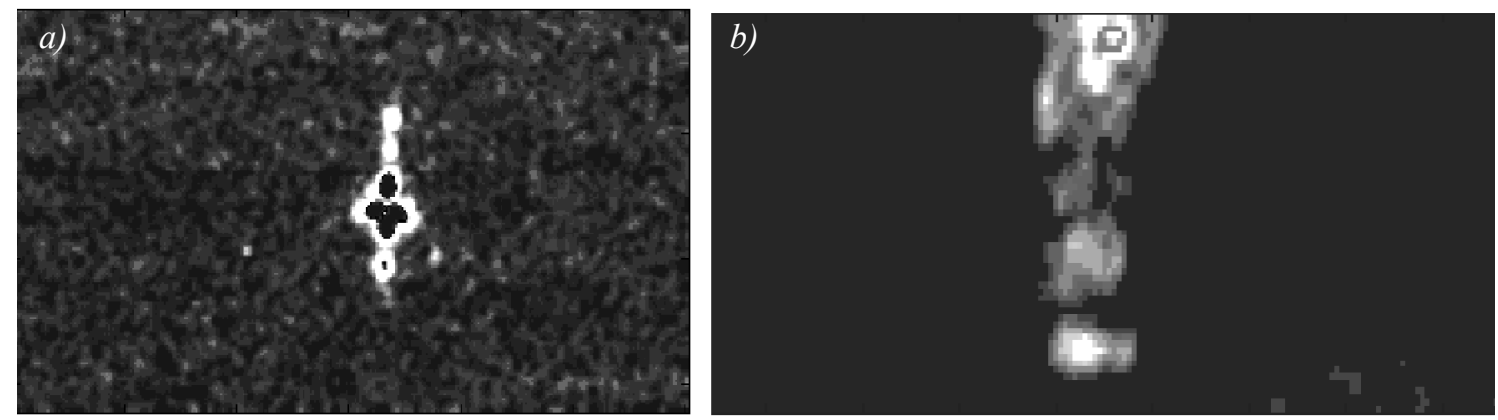

Fig. 26. NACE imaging in steel specimens (40 kHz-excitation): (9th to $11^{\text {th }}$ ) harmonic imaging of $50 \mu m$ a) wide fatigue crack, b) $(5 \times 40 \mathrm{~mm})$ hammer peening area in steel plate

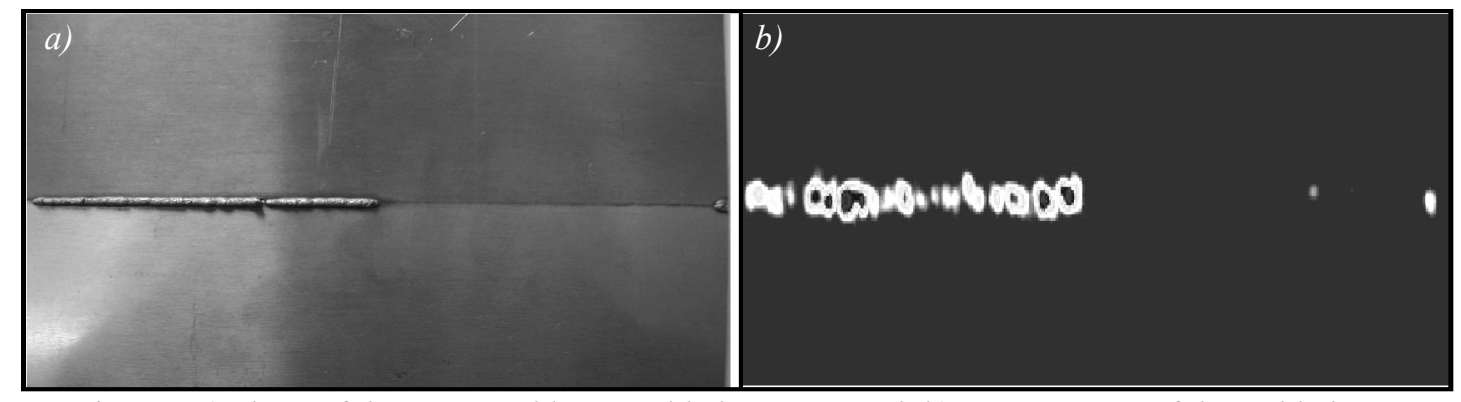

Fig. 27. a) photo of the measured laser welded joint in steel, b) NACE image of the welded join 
The feasibility of NACE by nonlinear defects is illustrated in Fig. 23a, which shows the third harmonic airborne radiation field from the delamination area in the "smart structure" specimen for piezo-excitation at $50 \mathrm{kHz}$ and 20 Vrms input. The image obtained by using air-coupled vibrometry [13] indicates that the source of nonlinear radiation is strictly confined in the defect area and NACE exhibits an evident directivity pattern.

The practical version of the NACE capable of locating and imaging the defects uses a highfrequency focused air-coupled (AC-) ultrasonic transducer as a receiver (Fig. 23b) [14]. Its bandpass frequency response combined with high-pass filtering circuit provides a strong rejection of the low-frequency excitation signal. The altitude angle varies to match the radiation directivity pattern. The nonlinear spectral components are then used as an input to AC-scanning equipment (AirTech 4000) for computer imaging of the NACE amplitude distribution over a specimen surface (C-scan).

In the experiments, a pair of focused (focus spot $\sim 2$ to $3 \mathrm{~mm}$, focus distance $40 \mathrm{~mm}$ ) AC-transducers with frequency responses centred at $\sim 400 \mathrm{kHz}$ and $\sim 450 \mathrm{kHz}$ ( $3 \mathrm{~dB}-$ bandwidth of $\sim 20 \mathrm{kHz}$ ) were alternately used as receivers in the $\mathrm{C}$-scan mode. Two excitation frequencies around 40 and $20 \mathrm{kHz}$ were used to maximize the NACE around (9th to $\left.11^{\text {th }}\right)$ and (23rd to $24^{\text {th }}$ ) higher harmonics, respectively.

Unlike NLV, which analyzes the light reflected from the specimen, the NACE-imaging relies on the direct nonlinear acoustic radiation by the defects. For the weakly-focused ACtransducers with cm-range depth of focus, the receiver is insensitive to medium scale variations of the surface profile. Our experiments show that NACE operates well in various constructional materials (wood, concrete, metals) with raw surfaces and rugged defects in components, like bolted joints, arc welds, etc.

In Fig. 24, the NACE imaging results are compared with NLV of multiple impact damage on the rear surface of a carbon fibre-reinforced (CFR-) multi-ply $\left(+45^{\circ} ;-45^{\circ}\right)$ composite plate $(175 \times 100 \times 1 \mathrm{~mm})$. Both techniques reliably visualize the defects with similar sensitivity.
The high lateral resolution of NACE is illustrated in Fig. 25 which shows a photo (a) of (5 $\mathrm{x} 15 \mathrm{~mm}$ ) delamination of specific shape in multiply GFRP plate. The mm-size contour details are reproduced closely in (9th to $\left.11^{\text {th }}\right)$ NACE image (right).

Fig. 26a shows the (9th to $\left.11^{\text {th }}\right)$ harmonic NACE image of a $50 \mu \mathrm{m}$-wide fatigue crack in a steel plate $(150 \times 75 \times 5 \mathrm{~mm})$ with two horizontally located grip holes for cyclic loading at some distance from the crack. The image reveals that the NACE detects not only the crack itself but also the fatigue structural damage in the plasticity areas between the crack and the grip holes.

To verify the NACE sensitivity to microdamage induced by plastic deformation, the NACE inspection was implemented for a steel specimen with a cold work area $(5 \times 40 \mathrm{~mm})$ produced by hammer peening. The image in Fig. 26b confirms that the NACE develops even without serious cracked defects and clearly discerns the microdamage induced by plastic deformation.

The NACE NDE-application was found to be particularly beneficial in metallic components where low acoustic damping facilitates the formation of standing waves, which produce a strong spurious background in the NLV. In particular, the images in Fig. 27 show that the NACE pattern reproduces well the quality of a laser weld-line between two steel components.

\section{SUMMARY}

The interaction of an ultrasonic wave with fractured defects is accompanied by frequency conversion, which is determined by nonlinear contact dynamics and strongly depends on the amplitude of the acoustic wave. At moderate driving amplitude, the CAN suggests a fully deterministic scenario with higher harmonic generation and/or wave modulation. These effects feature anomalously high efficiency, specific dynamic characteristics, modulated spectra, and unconventional waveform distortion. For the higher excitation, the family of nonlinear contact phenomena includes generation of ultrasubharmonics and frequency pair components, hysteresis, instabilities, transition to chaos, etc. 
that are well known in other branches of nonlinear physics.

The intact parts of the material outside the defect vibrate linearly, i.e. with no frequency variation in the output spectrum. Thus, in NNDT, a small cracked defect (transparent in the linear ultrasonic NDT) behaves as an active radiation source of new frequency components rather than a passive scatterer in conventional (linear) ultrasonic testing. This makes NNDT a unique defectselective instrument for localising and imaging of nonlinear flaws. The latter include a numerous class of contact defects, scaled from dislocations (nano-scale) to fatigue (micro-) cracks and macro-debonds in joints. Since the micro-contact (nonlinear) defects are only the forerunners of further major damage, the NNDT is thus capable of early recognition of material degradation and "predicting" the oncoming fracture.

Numerous case studies demonstrate the NNDE and defect-selective imaging by using scanning NLV and NACE. Particularly successful examples include hi-tech and constructional materials: impact damage and delaminations in fibre-reinforced plastics, fatigue micro-cracking and cold work in metals, delaminations in fibrereinforced metal laminates and concrete. Multiple nonlinear frequency components generated in imperfect materials provide abundant information on the properties and location of defects.

\section{REFERENCES}

[1] Solodov, I.Y. (1998). Ultrasonics of nonlinear contacts: propagation, reflection and NDE-applications. Ultrasonics, vol. 36, no. 1-5, p. 383-390.

[2] Solodov, I., Wackerl, J., Pfleiderer, K., Busse, G. (2004). Nonlinear self-modulation and subharmonic acoustic spectroscopy for damage detection and location. Appl. Phys. Lett., vol. 84, no. 26, p. 5386-5388, doi:10.1063/1.1767283.

[3] Solodov, I.Y., Krohn, N., Busse, G. (2002). CAN: An example of nonclassical acoustic nonlinearity in solids. Ultrasonics, vol. 40, no. 1-8, p. 621-625.

[4] Pecorary, C., Solodov, I. (2006). Nonclassical nonlinear dynamics of solid interfaces in partial contact for NDE applications. Universality of Non-Classical Nonlinearity with Application to NDE and Ultrasonics, Delsanto, P. (ed.), Chapter 19, p. 307-324, Springer Verlag, New York.

[5] Pfleiderer, K. (2006). Frequenzkonversion aufgrund nichtlinearer akustischer Phänomene: Grundlagen und Anwendung zur defektselektiven zerstörungsfreien Prüfung. Ph.D. Thesis, Stuttgart University, Sttutgart.

[6] Krohn, N., Pfleiderer, K., Solodov, I., Busse, G. (2002). Rev. Progress, QNDE, Thompson, D.O., Chimenti, D. (eds.) vol. 22, p. 981-988.

[7] Solodov, I., Pfleiderer, K., Busse, G. (2004). Nondestructive characterization of wood by monitoring of local elastic anisotropy and dynamic nonlinearity. Holzforschung, vol. 58 , no. 3 , p. 504-510.

[8] Solodov, I., Korshak, B. (2002). Instability, chaos, and "memory" in acoustic wavecrack interaction. Phys. Rev. Lett., vol. 88, 014303, p. 1-3.

[9] Minorsky, N. (1962). Nonlinear Oscillations. D. Van Nostrand Co. Inc., Princeton.

[10] Solodov, I., Pfleiderer, K., Busse, G. (2006). Nonlinear acoustic NDE: inherent potential of complete non-classical spectra. Universality of Non-Classical Nonlinearity with Application to NDE and Ultrasonics, Delsanto, P. (ed.), Chapter 29, p. 465-484, Springer Verlag, New York.

[11] Solodov, I., Pfleiderer, K., Busse, G. (2006). Nonlinear acoustic NDE using complete non-classical spectrum. Innovations in Nonlinear Acoustics, 17th ISNA, AIP, p. 35-

[12] Solodov, I., Busse, G. (2007). Nonlinear aircoupled emission: the signature to reveal and image micro-damage in solid materials. Appl. Phys. Lett., vol. 91, vol. 91, 251910, 2007.

[13] Solodov, I., Döring, D., Busse, G. (2009). Air-coupled laser vibrometry: analysis and applications. Appl. Optics, vol. 48, no. 7, p. C33-C37.

[14] Solodov, I., Busse, G. (2008). Listening for nonlinear defects: A new methodology for nonlinear NDE. Nonlinear Acoustics Fundamentals and Applications, 18th ISNA, AIP, p. 569-573. 